\title{
A Study of the Waves Supported by a Warm Plasma Slab
}

\author{
P. R. Caron \\ Aerospace Corporation, Los Angeles, Calif.
}

(Received November 13, 1964; revised December 14, 1964)

\begin{abstract}
The waves supported by a warm plasma slab are investigated. The transverse resonance equation describing the modes on the slab is obtained and, for certain ranges of plasma frequency, excitation frequency and plasma temperature, it is shown to be convenient to find the roots of this equation by the intersection of two loci. Using these loci the surface waves and leaky waves supported by the slab are described.
\end{abstract}

\section{Introduction}

A plasma slab serves as an appropriate idealized model for many practical situations of interest involving wave propagation through ionized layers. In particular, the re-entry communication problem is concerned with such a slab. This paper investigates the waves supported by a plasma slab which has finite temperature and no d-c magnetic field. Under these conditions a closed set of hydrodynamic equations can be written which, together with Maxwell's equations, indicate that two waves, viz, transverse and longitudinal, propagate independently in the plasma and couple only at the boundaries of a finite plasma region. Such a description is valid for wavelengths which are large by comparison with the Debye length in the plasma. When this condition is not satisfied a treatment using the linearized Boltzman equation is required. The problem of a line source near a semi-infinite plasma described by the hydrodynamic approximation has been solved [Hessel et al., 1962] and the coupling between the two wave types has been discussed.

The dispersion equation for the plasma slab is easily written. The zeros of this equation describe the waves supported by the plasma slab. For certain ranges of the ratio of plasma to excitation frequency, electron temperature and slab thickness, many roots of the determinant are found. These roots describe the propagation constants of the waves and it is found convenient to locate these roots by the intersection of two loci. The loci are easily characterized, and with their use, the variation of the propagation constants with the pertinent parameters is clearly displayed.

These same loci are also obtained from the transmission line analog of the problem by a suitable approximation, and it is shown that the waves supported by the slab are approximately determined by the transverse resonance of the plasma wave. The transmission line analog also shows that the modes can be subclassified into modes with even or odd transverse field variations. The same techniques are used to investigate damped modes on the slab, that is, modes which contribute to the radiation field. These are usually called "leaky waves."

\section{Dispersion Equation}

It is a simple matter to obtain the dispersion equation for the waves supported by a warm plasma slab. The TM waves, which are the waves of most interest, may be excited by a magnetic line source near the slab. Figure 1 shows such a geometry. The formal procedure [Caron and Stewart, 1964] involves writing down the wave equations for the longitudinal (plasma) and transverse (electromagnetic) waves in the slab, solving these to unknown coefficients and then using the boundary conditions which couple these waves to obtain a set of linear equations in the unknown coefficients. The dispersion equation is obtained by the requirement that the determinant of these equations be zero.

The dispersion equation can also be obtained via a transmission line analog and this analog will later be of help in the discussion of the waves supported by the slab. This analog is obtained by defining voltages and currents related to the transverse and longitudinal fields and by expressing the boundary conditions in terms of these new quantities [Hessel et al., 1962; Caron and Stewart, 1964]. The resulting analog is represented by figure 2 . In this figure,

$$
\begin{aligned}
& N=\frac{\omega_{p}}{\omega} \frac{k_{x}}{k_{0}} \frac{1}{\left(1-\omega_{p}^{2} / \omega^{2}\right)} . \\
& Z_{0}=K_{0} / \omega \epsilon_{0},
\end{aligned}
$$

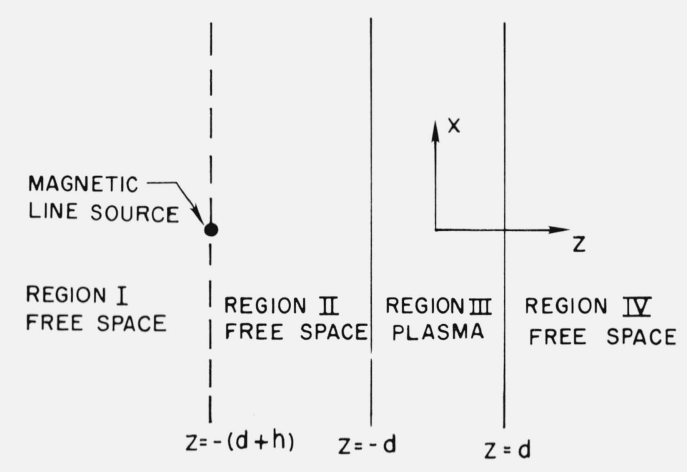

FiguRE 1. Geometry of the problem. 


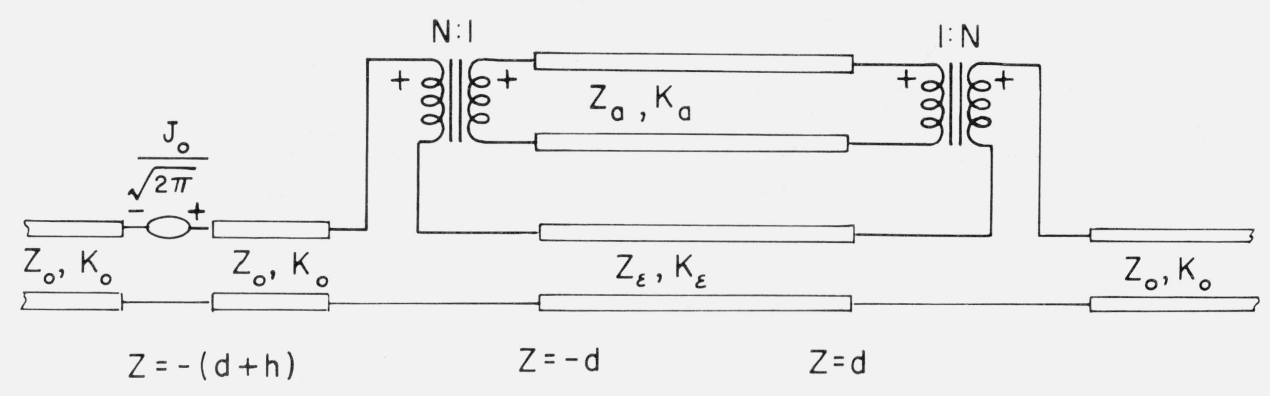

FIGURE 2. Schematic of the transmission line analog.

$$
\begin{aligned}
& Z_{\epsilon}=K_{\epsilon} / \omega \epsilon, \\
& Z_{a}=\frac{\mu_{0} \omega\left(1-\omega_{p}^{2} / \omega^{2}\right)}{K_{a}},
\end{aligned}
$$

where

$\omega_{p}^{2}=\frac{n_{0} e^{2}}{m \epsilon_{0}}$,

$k_{0}^{2}=\omega^{2} \mu_{0} \epsilon_{0}-k_{x}^{2}=k_{0}^{2}-k_{x}^{2}$,

$K_{\epsilon}^{2}=\omega^{2} \mu_{0} \epsilon_{0}\left(1-\omega_{p}^{2} / \omega^{2}\right)-k_{x}^{2}=\omega^{2} \mu_{0} \epsilon-k_{x}^{2}=k_{\epsilon}^{2}-k_{x}^{2}$,

$K_{a}^{2}=\omega^{2} / a^{2}\left(1-\omega_{p}^{2} / \omega^{2}\right)-k_{x}^{2}=k_{a}^{2}-k_{x}^{2}$,

$a$ is the electron sound speed and $k_{x}$ is the propagation constant along the slab. Certain features such as the independence of the longitudinal and transverse modes in the body of the plasma with coupling only at the boundaries are easily seen to be incorporated into the analog. The coupling mechanism is associated with the transformer of turn ratio $N: 1$ as well as with the impedance of each transmission line. This analog will later be used in discussing the waves supported by the slab.

Using the formal procedure the following system determinant is obtained:

$$
\Delta=8\left(\frac{\omega \epsilon_{0}}{n_{0} \epsilon}\right)^{2} e^{2 i K_{0} h}\left\{-2\left(\omega_{p}^{2} / \omega^{2}\right) K_{\mathrm{a}} K_{\epsilon}\right.
$$

$\left(1-\cos 2 K_{\epsilon} d \cos 2 K_{a} d\right)$

$$
-\left[\left(\omega_{p}^{2} / \omega^{2}\right)^{2} k_{x}^{2}+K_{a}^{2} / k_{x}^{2}\left\{K_{\epsilon}^{2}+K_{0}^{2}\left(1-\omega_{p}^{2} / \omega^{2}\right)\right\}\right]
$$

$\sin 2 K_{\epsilon} d \sin 2 K_{a} d+2 i\left(1-\omega_{p}^{2} / \omega^{2}\right) K_{0} K_{a}$

$$
\left.\begin{array}{l}
{\left[\frac{K_{a} K_{\epsilon}}{k_{x}^{2}} \cos 2 K_{\epsilon} d \sin 2 K_{a} d\right.} \\
\left.+\left(\omega_{p}^{2} / \omega^{2}\right) \sin 2 K_{\epsilon} d \cos 2 K_{a} d\right]
\end{array}\right\}
$$

It is easily argued that no surface waves exist on the real $\xi$ axis for $-1 \leqslant \xi \leqslant 1$. Thus all surface waves are slow waves. However, it will later be shown that complex poles occur very near to this section of the

The roots found by setting, $\Delta=0$, determine the propagation constants of the modes supported by the warm plasma slab. The roots with $k_{x}$ real describe surface waves whereas if $k_{x}$ is complex the roots describe what are generally termed "leaky waves." The equation for the roots can be written in the convenient form

$$
\begin{aligned}
\Delta=L\left\{-2 X \xi^{2}\left(1-C_{a} C_{\epsilon}\right)-\left[X^{2} \xi^{4}+\beta_{a}^{2}\left\{\beta_{\epsilon}^{2}\right.\right.\right. \\
\left.\left.+\left(1-\xi^{2}\right)(1-X)\right\}\right] S_{a} S_{\epsilon}+2 i(1-X) \\
\left.\sqrt{1-\xi^{2}}\left[X^{2} \xi^{2} S \epsilon C_{a}+\beta_{a}^{2} S_{a} C \epsilon\right]\right\}=0,
\end{aligned}
$$

where

$$
\begin{aligned}
L & =8 k_{0}^{2}\left(\frac{\omega \epsilon_{0}}{n_{0} e}\right) e^{2 j k_{0} h} \beta_{a} \beta_{\epsilon}, \\
X & =\omega_{p}^{2} / \omega^{2}, \\
\xi & =k_{x} / k_{0}, \\
\beta_{\epsilon} & =\sqrt{1-X-\xi^{2}}, \\
\beta_{a} & =\sqrt{\gamma^{2}-\xi^{2}}, \\
S_{a} & =\frac{\sin \alpha \beta_{a}}{\beta_{a}}, \\
S_{\epsilon} & =\frac{\sin \alpha \beta_{\epsilon}}{\beta_{\epsilon}}, \\
C_{a} & =\cos \alpha \beta_{a}, \\
C_{\epsilon} & =\cos \alpha \beta_{\epsilon}, \\
\gamma^{2} & =\left(c^{2} / a^{2}\right)(1-X), \\
\alpha & =2 k_{0} d .
\end{aligned}
$$


real axis. For $|\xi|>1$, interesting surface waves allied with warm plasma effects occur only for small values of $\gamma^{2}$. Since in general $c^{2} / a^{2} \gg 1$, this requires $X$ to be very near one. We therefore assume

$$
1-X \ll 1
$$

which, to a good approximation, yields the following expression for the location of the roots of $\Delta$ :

$2 \xi^{2}\left[1-\cos \left(\alpha \sqrt{\gamma^{2}-\xi^{2}}\right) \cosh (\alpha \xi)\right]$

$$
\begin{aligned}
& +\xi^{2}\left[\xi^{2}-\left(\gamma^{2}-\xi^{2}\right)\right] \\
& \frac{\sin \left(\alpha \sqrt{\gamma^{2}-\xi^{2}}\right)}{\sqrt{\gamma^{2}-\xi^{2}}} \frac{\sinh (\alpha \xi)}{\xi}=0 .
\end{aligned}
$$

With the definitions

$$
\begin{aligned}
& z=\alpha \xi \\
& \eta^{2}=\alpha^{2} \gamma^{2}, \\
& w^{2}=\eta^{2}-z^{2},
\end{aligned}
$$

it can be shown that the zeros of (12) may be located by the intersections of the following two loci in the $w, z$ plane:

$$
\begin{gathered}
1-\cos w \cosh z=\frac{1}{2 z w}\left(w^{2}-z^{2}\right) \sin w \sinh z, \\
w^{2}+z^{2}=\eta^{2} .
\end{gathered}
$$

In this $w, z$ plane (13) appears as shown in figure 3 . Thus the locus consists of an infinite number of branches ${ }^{1}$ which for large $z$ are asymptotic to $w$ $=n \pi, n=1,2,3$... The intersections of these branches with the circle described by (14) give the values of $z$ and hence $k_{x}$ which define the propagation constants for the surface waves.

'Note that only the first six branches are shown in figure 3.

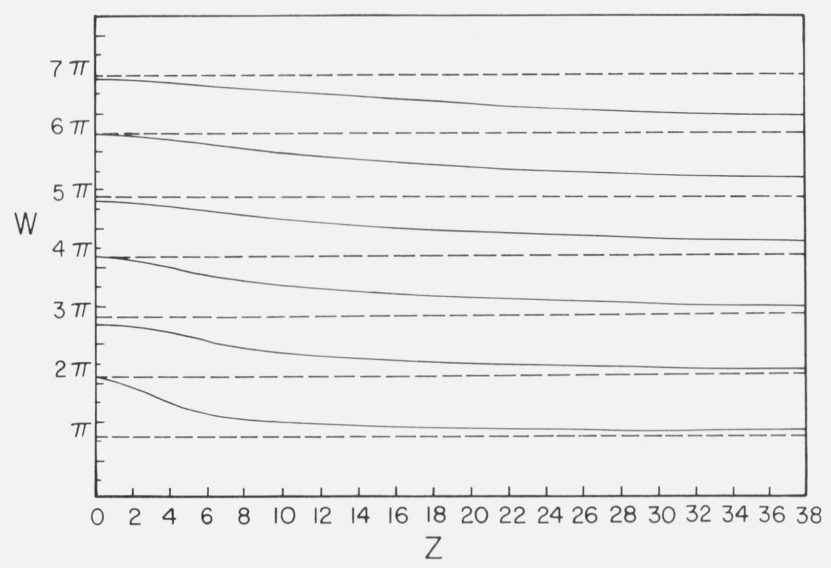

FigURE 3. Locus in the $\mathrm{w}, \mathrm{z}$ plane used to locate surface wave poles and complex poles.

\section{Discussion of Surface and Leaky Waves}

Figure 3 can be used to discuss some properties of the surface waves. For the special case $\gamma^{2}=1$ so that $\eta=\alpha$, it is apparent that as $\alpha$ increases from zero, no surface waves exist until $\alpha$ approaches $2 \pi$, that is, until the slab thickness approaches the free space wavelength, which for $\gamma^{2}=1$ equals the wavelength of the plasma mode. For other values of $\gamma^{2}$ it is clear that surface waves do not exist unless the slab thickness is near the wavelength of the plasma wave. Clearly, the surface waves are like guided acoustic waves with some anomalous behavior which will not be discussed.

It turns out that as $\alpha \gamma \longrightarrow 2 \pi$, two surface waves exist. That is, the first branch of the locus in figure 3 intersects the circular locus of (14) twice for $\alpha \gamma \leqslant 2 \pi$. These intersections will later be shown to describe a backward and a forward wave. For $\alpha \gamma>2 \pi$ but not in the vicinity of $3 \pi$ there is only one surface wave which, as will be shown later, is a forward wave. Near $\alpha \gamma=3$ we are concerned with the intersection of the second branch of figure 3 with the circle of radius $\alpha \gamma$. This branch has a $w$ intercept which is less than $3 \pi$ and never intersects the circular locus more than once. Furthermore, this intersection describes a forward wave. As $\alpha \gamma \longrightarrow 2 n \pi,(n=2,3,4 \ldots)$, and as $\alpha \gamma \longrightarrow 2(n+1 / 2) \pi,(n=2,3,4 \ldots)$, the behavior is quantitatively like the respective behavior for $n=1$. Therefore, the surface waves are much like guided acoustic waves but the coupling of these waves to the electromagnetic fields both inside and outside the plasma slab produces certain anomalies like:

(1) no resonance appears for $\alpha \gamma$ near $\pi$;

(2) near $\alpha \gamma=2 n \pi,(n=1,2,3 \ldots)$, there are two surface waves, one forward and one backward;

(3) near $\alpha \gamma=2(n+1 / 2) \pi,(n=1,2,3 \ldots)$, the surface wave exists for the slab thickness slightly less than an integral number of acoustic wavelengths.

More insight into the behavior of these surface waves may be obtained by using the transmission line analog shown schematically in figure 2 . Under the assumptions used in this paper, it is clear that $Z_{\epsilon}$ is imaginary, i.e., reactive. Also it is easily shown that the sum of this impedance plus the impedance of the plasma transmission line reflected through the transformer is much greater in magnitude than the free space line impedance except when this sum is very near the resonances. Therefore, to a good approximation, the resonances can be found by considering the symmetrical circuit consisting of the plasma line terminated by a purely imaginary load at each end. It is evident that the resonances can then be described in terms of even and odd modes on the line. With the use of elementary transmission line theory and the definition of $z, \eta$, and $w$ given previously, the resonance conditions are represented by the intersection of the loci,

$$
\begin{aligned}
& z \tanh (z / 2)+w \tan (\mathrm{w} / 2)=0, \text { even modes, } \\
& z \operatorname{coth}(z / 2)-w \cot (\mathrm{w} / 2)=0, \text { odd modes, }
\end{aligned}
$$


with the circular locus, $w^{2}+z^{2}=\eta^{2}$. It is a straightforward matter to show that (15a) and (15b) are equivalent to (13). However, these equations show that the branches of figure 3 are subdivided into two sets even modes and odd modes-and these sets correspond to the cases previously considered where resonance occurred for $\alpha \gamma$ near $2 n \pi,(n=1,2,3$. . .), and near $2(n+1 / 2) \pi,(n=1,2,3 \ldots)$, respectively. Therefore, the different characteristics of these cases must be allied with the even and odd character of the modes. The neglect of the free space line impedance is equivalent to the neglect of the terms in (10) with the factor $(1-X)$.

We shall now determine whether the surface waves are forward or backward waves. The phase velocity of these waves along the slab is $v_{x p}=\omega / k_{x}$ and the group velocity is $v_{x g}=d \omega / d k_{x}$. Therefore, the wave is forward or backward if $v_{x p}$ and $v_{x g}$ have the same or the opposite sign respectively. We assume the phase velocity to be positive and investigate the sign of

$$
\frac{d k_{x}}{d \omega}=\frac{d k_{x}}{d \eta} \frac{d \eta}{d \omega}=\frac{d k_{x}}{d \eta}\left(\gamma \frac{d \alpha}{d \omega}+\alpha \frac{d \gamma}{d \omega}\right)
$$

For the assumptions of this paper it is not difficult to show that

$$
\alpha \frac{d \gamma}{d \omega} \gg \gamma \frac{d \alpha}{d \omega}
$$

and using (1lj) along with the definitions of $z$ and $\eta$,

$$
\frac{d k_{x}}{d \omega}=\frac{1}{a} \frac{1}{\sqrt{1-X}} \frac{d z}{d \eta} .
$$

Therefore, if $d z / d \eta$ is positive or negative the wave is forward or backward. From the previous discussion backward waves exist only when $\alpha \leqslant 2 n \pi$. For this condition there are two intersections of the loci in the $w, z$ plane and the backward wave is determined by the intersection at smaller $z$.

The reader has probably noticed that for certain values of $\eta=\alpha \gamma$ some of the intersections of the locus of figure 3 with the circular locus, $w^{2}+z^{2}=\eta^{2}$, occur in the range $0 \leqslant \xi \leqslant 1$, which is contradictory to previous statements. However, inspection of (10) shows that the neglected terms containing $(1-X)$ will move these poles off the real axis in the $\xi$ plane. On the other hand, the poles for which $\xi>1$ are displaced along the real axis by these terms. This behavior is even more clearly visualized via the transmission line analog. With this analog the resonances were obtained by neglecting the coupling to the free space lines, and from the expression for $Z_{0}$, the characteristic impedance of these lines, it is clear that $Z_{0}$ is real or imaginary when $\xi<1$ or $\xi>1$. Hence, for $\xi<1$ the free space lines will contribute a small real impedance to the otherwise reactive impedance terminating the plasma transmission line whereas for $\xi>1$ the contribution will be a small additional reactance. Therefore, to a good approximation, the analysis used so far completely describes the surface and leaky waves except for the small attenuation factor of the leaky waves as they propagate along the slab.

A general case is depicted in figure 4 where $\gamma^{2}>1$ has been assumed. Since $z=\alpha \xi$, the roots $z_{1}$ and $z_{2}$ correspond to leaky waves whereas the roots $z_{3}$ and $z_{4}$ represent surface waves. One notes that for $\gamma^{2}=1$ all the roots represent leaky waves and as $\gamma^{2}$ increases from one with fixed $\alpha$ more and more roots are obtained but now the roots are divided between those which determine leaky waves and those which determine surface waves. This phenomenon can be explained in terms of the optics of the various waves. For $\gamma=1$, the propagation constant of the plasma wave equals the free space constant, and therefore, the plasma wave couples to propagating electromagnetic waves at the two interfaces and hence energy leaks

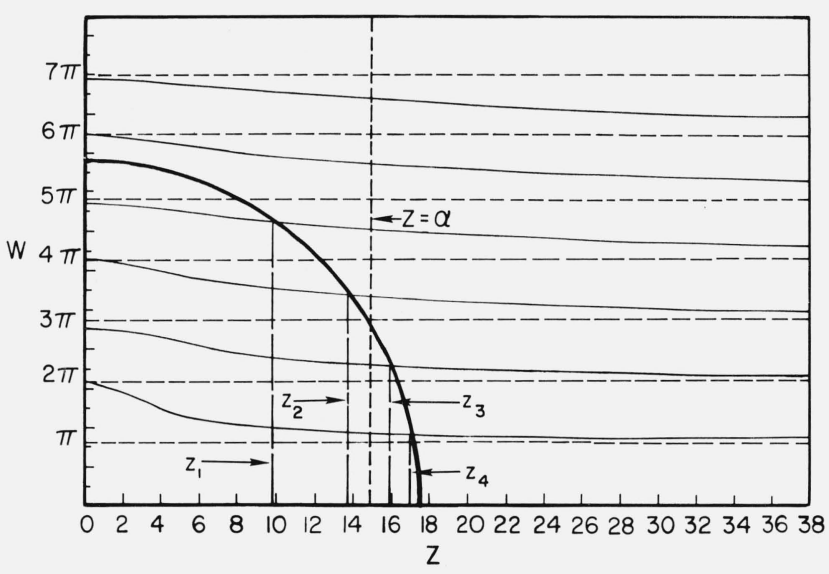

FiguRE 4. Example of the location of surface wave poles and complex poles using the loci in the $\mathrm{w}, \mathrm{z}$ plane.

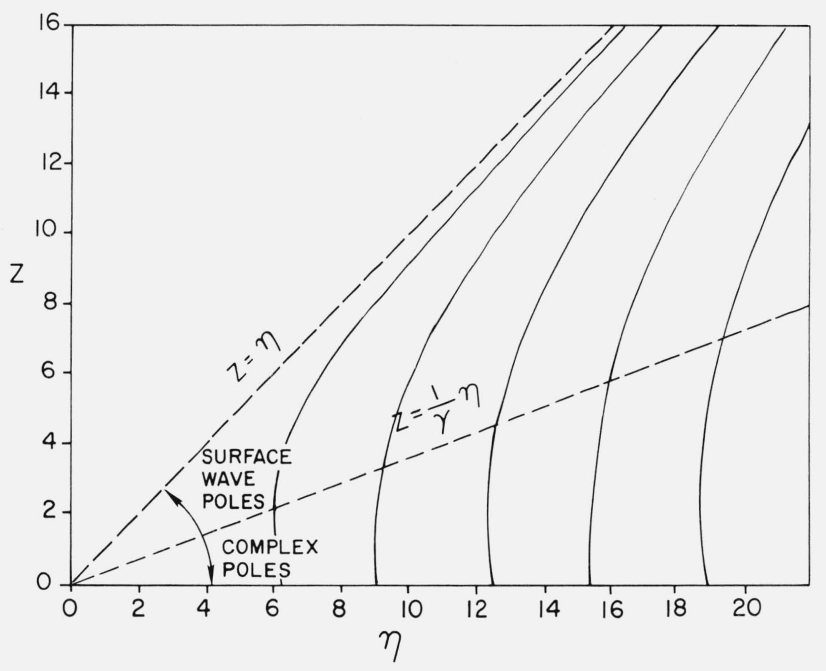

FIGURE 5. Dispersion curves: $\mathrm{z}$ versus $\eta$. 


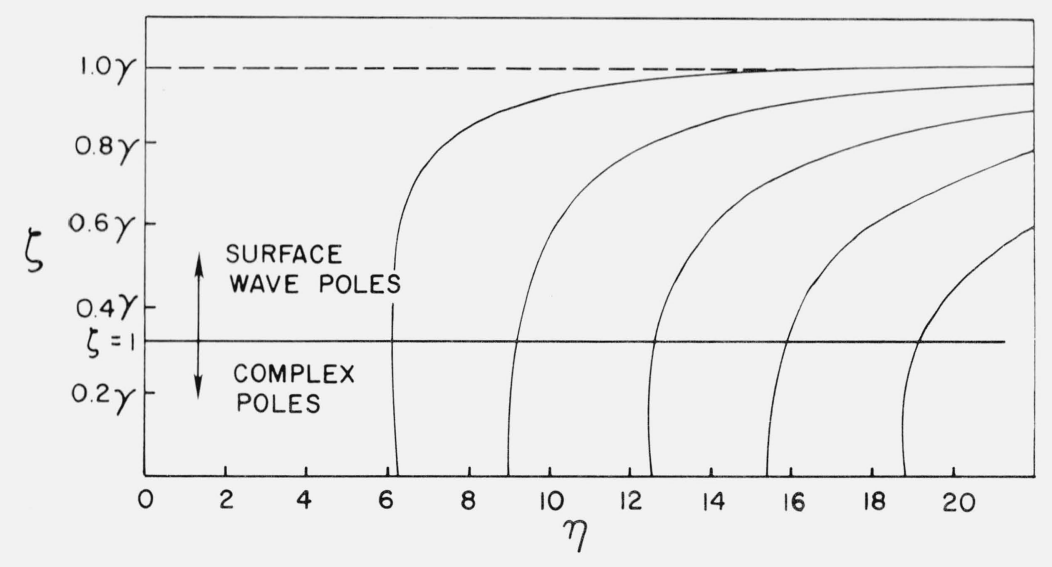

FiguRE 6. Dispersion curves: $\zeta$ versus $\eta$.

from the slab. Conversely, for $\gamma^{2}>1$, the transmission angle of the electromagnetic waves at each interface is greater than the incidence angle of the plasma wave and therefore the plasma waves with the largest incidence angles may lead to complex transmission angles or attenuating electromagnetic waves thus defining a surface wave. Similar behavior occurs for $\gamma^{2}<1$ but in this case, the plasma wave is also allowed to attentuate in the transverse direction. The previous discussion of the backward and forward nature of the waves also holds in an approximate sense for the leaky waves but now the waves have a small attenuating factor in the direction of energy propagation.

It is useful at this point to draw some "dispersion" curves for the surface and complex waves. Using the locus of figure 3 and the circular locus of radius $\eta$, the curves shown in figure 5 giving $z$ as a function of $\eta$ are drawn. These are called "dispersion" curves for the modes represented by the various branches. It is also of use to draw corresponding curves of $\xi$, the normalized propagation factor along the slab, versus $\eta$ and these are shown in figure 6 . In these two figures, $\gamma^{2}>1$ has been assumed. Some of the points already discussed such as the existence of both a forward and a backward wave for certain values of $\eta$ are even more clearly understood from figures 5 and 6 .

\section{References}

Caron, P. R., and G. E. Stewart (July 1964), Radiation from a line source in a ground plane covered by a warm plasma slab, Aerospace Corporation Report No. ATN-64(9230)-3.

Hessel, A., N. Marcuvitz, and J. Schmoys (Jan. 1962), Scattering and guided waves at an interface between air and a compressible plasma, IRE Trans. Ant. Prop. AP-1 0, No. 1, 48-54.

(Paper 69D5-508) 